\title{
BMJ Open Influenza-like illness and antimicrobial prescribing in Australian general practice from 2015 to 2017: a national longitudinal study using the MedicineInsight dataset
}

Carla De Oliveira Bernardo,, ${ }^{\oplus 1}$ David Gonzalez-Chica, ${ }^{\circledR 1,2}$ Nigel Stocks ${ }^{๑ 1,3}$

To cite: Bernardo CDO, Gonzalez-Chica D, Stocks N. Influenza-like illness and antimicrobial prescribing in Australian general practice from 2015 to 2017: a national longitudinal study using the Medicinelnsight dataset. BMJ Open 2019;9:e026396. doi:10.1136/ bmjopen-2018-026396

- Prepublication history and additional material for this paper are available online. To view these files, please visit the journal online (http://dx.doi. org/10.1136/bmjopen-2018026396).

Received 30 August 2018 Revised 22 January 2019 Accepted 27 March 2019

Check for updates

(C) Author(s) (or their employer(s)) 2019. Re-use permitted under CC BY-NC. No commercial re-use. See rights and permissions. Published by BMJ.

${ }^{1}$ Discipline of General Practice, Adelaide Medical School, University of Adelaide, Adelaide, South Australia, Australia

${ }^{2}$ Adelaide Rural Clinical School, University of Adelaide, Adelaide, South Australia, Australia

${ }^{3}$ Australian Partnership for

Preparedness Research on InfectiouS disease Emergencies (APPRISE), Adelaide, South Australia, Australia

Correspondence to

Dr Carla De Oliveira Bernardo; carla.bernardo@adelaide.edu.au

\section{ABSTRACT}

Objectives To investigate the epidemiology of influenza-like illness (ILI) by general practice and patient characteristics, and explore whether sociodemographic variables or comorbidities affect antiviral or antibiotic prescribing.

Design Open cohort study.

Setting A representative sample of 550 Australian general practices contributing data to the Medicinelnsight programme.

Participants 4228149 patients of all age groups who had at least one consultation between 2015 and 2017. Median age was 37 years (Interquartile range: 21-57), $54.4 \%$ women, $16.4 \%$ aged $\geq 65$ years, $2.2 \%$ Aboriginal or Torres Strait Islander, $17.6 \%$ had a chronic disease and $18.1 \%$ a mental health condition.

Primary and secondary outcome measures ILI consultation rates (per 1000 consultations) were calculated using all ILI diagnoses for all clinical encounters with a general practitioner between 2015 and 2017. Antiviral and antibiotic prescribing for ILI cases were investigated and logistic regression models adjusted for practice and patient characteristics used to analyse associations.

Results ILI consultation rates in 2017 were higher than in previous years. Antiviral prescribing increased from $20.6 \%$ in 2015 , to 23.7 in 2016 and $29.7 \%$ in 2017 , while antibiotic prescribing decreased from $30.3 \%$ to $28.0 \%$ and $26.7 \%$, respectively ( $p<0.05$ in both cases). Practices located in high socioeconomic areas had higher ILI consultation rates ( 4.3 vs 2.5 per 1000 consultations, $\mathrm{p}<0.05)$, antibiotic $(30.7 \%$ vs $23.4 \%, p<0.05)$ and antiviral $(34.2 \%$ vs $13.5 \%, \mathrm{p}<0.05)$ prescribing than those in lower socioeconomic areas. The coexistence of chronic or mental health conditions was associated with lower ILI consultation rates, higher antibiotic use, but not with antiviral prescribing. However, those with chronic respiratory conditions had a higher frequency of antibiotic and antiviral prescribing in 2017 than individuals with other comorbidities.

Conclusions Although the apparent decline in antibiotic prescribing for ILI is welcome, the increase in antiviral use may not be targeting those at high risk of complications from infection.
Strengths and limitations of this study

- A large sample: more than 4.2 million patients and 32 million consultations in Australian general practice over 3 years.

- Patients of all age groups, ethnicity or sex, and practices from all Australian states, socioeconomic areas and geographic regions.

- The study explores antimicrobial prescribing over time and their association with comorbidities.

- Although the data reflects real clinical practice with diagnoses and treatment decisions recorded by general practitioners (GPs), the quality and completeness of recording may vary (between GPs and on day-to-day basis depending on GPs workload), since the information could have been recorded as free text instead of a standardised medical code.

\section{INTRODUCTION}

Influenza is a viral infectious disease of the respiratory tract caused by influenza viruses. Although symptoms may be mild to moderate and last a few days, influenza can cause severe illness especially among high-risk groups, such as children (aged $<5$ years), the elderly, pregnant women and individuals with chronic or immunosuppressive conditions. The WHO estimates influenza is responsible for up to 650000 annual deaths worldwide. ${ }^{1}$ In Australia, influenza and pneumonia together represent the 11th leading cause of death. ${ }^{2}$ Therefore, appropriate management of influenza in primary healthcare settings for high risk groups is essential when treating more severe cases. ${ }^{34}$

For most individuals, influenza is usually self-limiting and only symptomatic management is necessary. ${ }^{1}$ However, antivirals have been recommended for the treatment of influenza among ambulatory patients as it shortens the length of the disease ${ }^{5}$ and seems 
to lessen symptoms and complications. ${ }^{6}$ Moreover, these medications reduce the likelihood of death by $25 \%$ among hospitalised individuals ${ }^{7}$ and although the use of prophylactic antivirals for contacts may decrease the risk of developing symptomatic influenza, their potential side effects hinder its recommendation as a preventive strategy. ${ }^{5}$ However, prescribing antibiotics for most acute uncomplicated respiratory infections, including influenza, are not recommended by guidelines, due to marginal effectiveness, risk of antibiotic resistance, common side effects and financial cost. ${ }^{8}$ In fact, antibiotic overprescribing can expose patients to severe adverse events and has been associated with up to 23000 deaths annually in the USA. ${ }^{10}$

Despite these recommendations, the prescription of antibiotics for uncomplicated acute respiratory infections remain high worldwide. ${ }^{11-13}$ In the USA, a study carried out during the 2013-2014 and 2014-2015 influenza seasons reported antibiotics were prescribed in $29 \%$ of influenza cases, while $20 \%$ received antivirals. ${ }^{12}$ Data collected in 2013-2015 from a large electronic medical record database in England showed that antibiotic prescribing was $18 \%$ among patients with influenza like illness (ILI) and no comorbidities and $28 \%$ among those with some comorbidity (same-day prescription). ${ }^{14}$ In Australia, the Bettering the Evaluation and Care of Health programme found that, between 2010 and 2015, 11\% of individuals with influenza were managed with antibiotics, and the rate was as high as $85 \%$ among patients with other acute respiratory infections. ${ }^{15}$ Nonetheless, there is little data about antiviral prescribing for influenza in Australian general practice, and there no contemporary descriptions of antibiotic use.

Therefore, this study aimed to investigate the epidemiology of ILI in Australian general practice between 2015 and 2017, describe ILI consultation rates (per 1000 consultations) by general practice and patient characteristics, and explore whether sociodemographic variables or the presence of comorbidities affect the prescription of antivirals or antibiotics.

\section{METHODS}

\section{Data source}

The study included data from MedicineInsight, a national general practice programme developed and managed by the National Prescribing Service (NPS) MedicineWise with funding support from the Australian Government Department of Health (the MedicineInsight programme is described at http://www.nps.org.au/medicine-insight). This programme extracts deidentified medical records from 656 general practices and more than 4.5 million patients, allowing the development of a large longitudinal database with patients of all ages, states and Australian regions, including practices varying by size, billing methods and type of services offered. Patients within each practice receive a unique identifying number before having their electronic medical records de-identified and securely transferred to NPS MedicineWise. Routinely collected data includes sociodemographic data (gender, ethnicity, year of birth, residential postcode), clinical data (diagnoses, reasons for consultation, immunisations), health measurements (temperature, blood pressure, weight, height, waist circumference, laboratory results) and prescribed medications. Details of the data collection process used by MedicineInsight have been published elsewhere. ${ }^{1617}$

\section{Sample selection}

This is an open cohort study including all patients who had at least one consultation between January 2015 and November 2017 within a MedicineInsight practice. Only practices established for at least 2 years before the analysis period and those that had no interruptions of 6 weeks or more in data extraction were included. Patients were excluded when the reason for encounter was suggestive of an administrative contact (eg, 'phone call', 'email', 'letter', 'filling forms', 'reminder'). Duplicated registers for the same patient on the same date were also excluded (ie, only one encounter per patient per day was preserved for analysis, with all information about reason for encounter, diagnosis and medication from both encounters used). Therefore, after applying these criteria, the total number of registered MedicineInsight encounters for the period 2015-2017 were reduced from 69361222 to 32254 306, representing 4228149 patients attending 550 general practices across Australia (online supplementary figure 1).

\section{Data extraction}

According to Australian guidelines, ${ }^{18}$ ILI is defined as the presence of fever, cough and fatigue, which are symptoms commonly seen among patients with influenza. However, as this study used secondary data that could include the diagnosis of ILI instead of their symptoms, a mixed strategy was used to identify all possible cases. Moreover, to improve data quality we searched for specific terms in different fields of the MedicineInsight database, including medical diagnosis recorded by the general practitioners (GPs), reasons for encounter, prescribed medications and clinical measurements.

Although GPs are encouraged to record patients' medical data by using medical vocabulary coding systems (ie, 'DOCLE', 'PYEFINCH'), its use is not compulsory and medical terms can be entered as free text. ${ }^{17}$ For that reason, synonyms of ILI (eg, 'influenza', 'flu-like illness', 'flu') or their symptoms (ie, defined as the concomitant report of fever, cough and fatigue) ${ }^{19}{ }^{20}$ were also included in the searching algorithm (including possible misspellings of these terms). The diagnosis of fever was also considered when the GP recorded a body temperature $\geq 38^{\circ} \mathrm{C}$ for that consultation (diagnosis, reason for encounter or clinical measurement fields). Furthermore, those consultations in which GPs prescribed some of the US Food and Drug Administration-approved anti-influenza medications (ie, oseltamivir, zanamivir, peramivir 
or their brand names Tamiflu, Relenza and Rapivab) were also coded as positives for ILI cases $(\mathrm{n}=6.922$ or $7 \%$ of all ILI cases; 2015=7\%; 2016=7\%; 2017=8\%; online supplementary figure 1), even though the diagnosis of ILI or their symptoms was not recorded as a diagnosis or reason for encounter, since previous studies found it commonly happens ${ }^{2122}$ and is a recommendation for quality improvement of studies based on routinely-collected health data. ${ }^{23-25}$ Given the natural life cycle of influenza infections, ${ }^{1}$ all new attendances for ILI by the same patient occurring within 14 days of the first ILI diagnosis were considered part of the same event instead of a new ILI episode.

Regarding medications, all patients with a diagnosis of ILI had their prescription history investigated to identify those who received a prescription for an antiviral and/or an antibiotic. The list of antibiotics included penicillins, tetracyclines, cephalosporins, sulphonamides, macrolides and quinolones. Topical antibiotics were excluded from this search. Individuals were considered to have received any of these medications for the management of ILI when (1) the medication was prescribed on the same date of the ILI diagnosis, or (2) it was prescribed within 14 days after the original ILI diagnosis AND no other reason for that prescription was identified in the database (any illness requiring the use of antibiotics, ${ }^{9}$ such as pneumonia, streptococcus infection, bacterial infection, chronic sinusitis, urinary tract infection or cholecystitis, triggered a negative categorisation for ILI treatment).

\section{Outcomes}

ILI consultation rates (or 'attack' consultation rate) were calculated using the number of ILI cases per 1000 consultations with the GP for any reason in a specific period (weekly and annually). In addition, percentages of ILI cases managed with antivirals or antibiotics were also estimated.

\section{Covariates}

Practice information included state (New South Wales; Victoria; Queensland; Western Australia; Tasmania; South Australia; Australian Capital Territory; Northern Territory), rurality (major cities; inner regional; outer regional/remote/very remote), and the Index of Relative Socioeconomic Advantage and Disadvantage (IRSAD) quintiles. ${ }^{26}$ IRSAD is a macroeconomic indicator of relative economic and social advantage/disadvantage position within an area compared with the rest of the country. ${ }^{26}$ Therefore, a higher IRSAD quintile indicates the practice is in a more advantaged area.

Patients' sociodemographic characteristics included gender (male; female), age (in years and categorised as: $<5$ years, $5-17,18-44,45-54$ and $\geq 65$ years), ethnicity (Aboriginal and/or Torres Strait Islander yes or no) and patient's IRSAD quintiles (based on their residential postcode).$^{26}$

Because the presence of some chronic comorbidities can influence antibiotic prescribing due to their higher risk of influenza complications, ${ }^{27}{ }^{28}$ some diseases were also extracted from the database and investigated as covariates. They included: (1) cardiac disease (ie, coronary artery disease, atrial fibrillation or heart failure); (2) chronic respiratory conditions (ie, cystic fibrosis, chronic obstructive pulmonary disease, chronic emphysema or severe asthma [those requiring the use of prednisolone for asthma management]); (3) chronic neurological conditions (ie, multiple sclerosis or spinal cord injuries); (4) immunocompromising conditions (ie, those with HIV infection or malignancy); (5) chronic liver disease (ie, liver fibrosis or cirrhosis) or (6) other relevant chronic illnesses (ie, diabetes mellitus, chronic renal failure or haemoglobinopathies). Individuals were considered as having a comorbidity when they were found to have one or more of these diseases. Finally, the diagnosis of a mental health condition (depression/anxiety) was also included as a covariate in this study, as they can also influence medication prescription. ${ }^{29} 30$

\section{Statistical analysis}

ILI consultation rates in each calendar week from 2015 to 2017 were calculated and the results presented graphically. Tables were used to show the annual ILI consultation rates according to practice and patient characteristics.

The association between practice characteristics and ILI consultation rates was assessed using logistic regression with robust standard errors (SEs), to account for clustering within practice, with all variables mutually adjusted. A similar procedure was used to evaluate the association between ILI consultation rates and patients' characteristics (sociodemographic and clinical). In this case, sociodemographic and clinical variables (comorbidities and mental health conditions) were adjusted for age, gender and characteristics of the practice (state, rurality and IRSAD quintiles).

We also used logistic regression models to analyse the determinants of antiviral and antibiotic prescription among patients with ILI, by practice and patient characteristics. Marginal adjusted probabilities of ILI diagnosis (rates per 1000 consultations) and medication use (percentages of antiviral or antibiotic prescribing for ILI cases) in each category of the exposure variables were estimated after the regression models, and pseudo $\mathrm{R}^{2}$ are presented to assess the magnitude of the association between the outcomes and practice and patient characteristics.

Logistic regression models were also used to test differences in ILI rates and medication use between 2015 and 2017 in each category of the explanatory variables. Results for the prescription of antivirals or antibiotics among patients with ILI according to the presence of some chronic disease (respiratory, cardiac, neurological or liver disease, immunocompromising conditions or other) was presented graphically, including their respective $95 \%$ confidence intervals $(95 \% \mathrm{CI})$.

All analyses were performed in the statistical software Stata V.15.0 (StataCorp). 


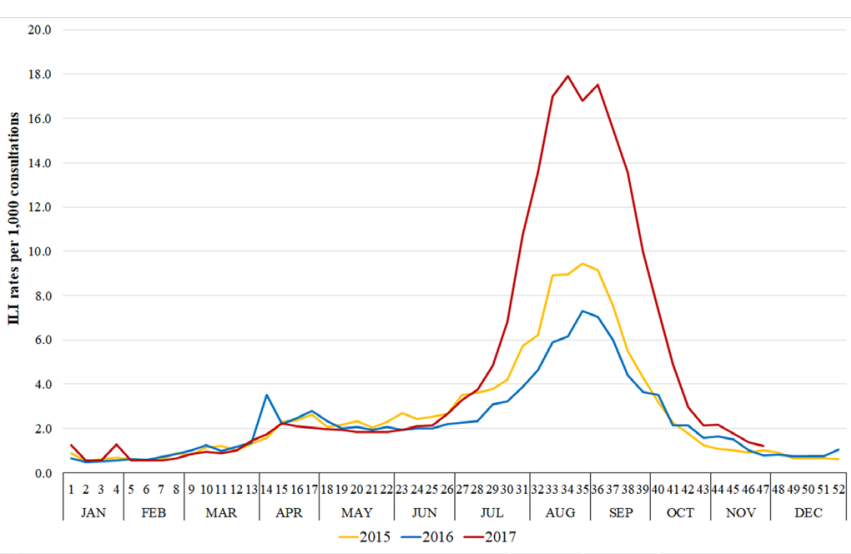

Figure 1 Weekly Influenza-like illness (ILI) rate per 1000 consultations in Australian general practices from 2015 to 2017.

\section{Patient and public involvement}

Patients and the public were not involved in the development of the research question or outcome measures, design of the study or conduct of the study.

\section{RESULTS}

The study includes results from 32254306 encounters (2015=11 581 786; 2016=12 009 848; 2017=8 662 672) and 4228149 patients, of all age groups, attending 550 general practices. Of these encounters, $61 \%$ occurred in major cities and $15 \%$ in outer regional/remote/very remote Australia, 23\% in areas with the highest IRSAD quintile and $21 \%$ in the bottom quintile (data not shown in tables). The median age of all patients in the dataset was 37 years (interquartile range (IQR): 21-57 years), $7 \%$ were children aged $<5$ years, $14 \%$ aged 5 to 18 years and $16 \%$ aged $\geq 65$ years, $54 \%$ were women, $2 \%$ Aboriginal or Torres Strait Islanders, $18 \%$ had an investigated comorbidity and $18 \%$ a mental health condition. Patients diagnosed with ILI (diagnosis in any year) had a slightly higher median age (40 years; IQR: 22-57) than the whole sample, $5 \%$ of them were children $<5$ years, $15 \%$ aged $5-17$ years, $16 \%$ aged $\geq 65$ years and the proportion of women $(55 \%)$ or Aboriginal or Torres Strait Islanders (2\%) was similar. The prevalence of comorbidities $(25 \%)$ and mental health conditions $(26 \%)$ were both higher among those with ILI.

\section{ILI consultation rates}

The total number of ILI cases for the period 2015-2017 was 98086 (ILI consultation rate=3.0 per 1000 consultations). The ILI consultation rate in 2017 (4.4 per 1000 consultations) was at least $63 \%$ higher (equivalent to an increase of 1.7 per 1000 consultations) than in previous years (2.7 and 2.3 per 1000 consultations in 2015 and 2016, respectively). The peak of ILI cases were observed between weeks 33th and 36th in every year (figure 1), with up to 18 cases per 1000 consultations.

Table 1 shows ILI consultation rates per 1000 consultations according to general practice and patient characteristics. ILI consultation rates were higher in the two biggest Australian states (New South Wales and Victoria) and Tasmania, while the Australian Capital Territory showed a consistently low ILI consultation rate over the period. Western Australia had the lowest ILI consultation rate in 2017. In 2015 and 2016, ILI consultation rates were quite similar among practices located in urban and rural areas, but in 2017 the rate was at least $32 \%$ higher in practices located in major cities. Finally, practices located in the top IRSAD quintile areas showed the highest ILI consultation rate in all years. Nevertheless, when comparing practices located in the top and bottom IRSAD quintiles, the relative difference in ILI consultation rates changed from $82 \%$ in 2015 to $67 \%$ in 2017.

\section{Antivirals and antibiotics prescribing}

Overall, $25.0 \%$ of the 98,086 ILI cases were managed with antivirals and $28.3 \%$ with antibiotics $(8.2 \%$ with both medications prescribed at the same time). The prescription of antivirals changed from $20.6 \%$ in 2015 , to $23.7 \%$ in 2016 and $29.7 \%$ in 2017 (table 2), while antibiotics prescribing changed from $30.3 \%$, to $28.0 \%$ and $26.7 \%$, respectively $(\mathrm{p}<0.05$ in both cases). Antiviral prescribing was at least twice as frequent among practices located in New South Wales or Victoria than in South Australia or Northern Territory, as well as among those located in top IRSAD quintile areas compared with those in the bottom quintile. On the other hand, antiviral prescribing was slightly lower among practices located in inner regional Australia compared with other areas.

Table 1 also shows ILI consultation rates were lower in women, individuals aged $<5$ years or $\geq 65$ years, and among Aboriginal or Torres Strait Islanders than their peers, and these differences remained steady over the years. Disparities in ILI consultation rates according to the individual's IRSAD quintile were less evident than for the practice's IRSAD. Finally, people with comorbidities or mental health issues showed lower ILI consultation rates than their counterparts $(40 \%$ and $20 \%$ less frequent, respectively), and this difference remained stable over time.

Table 2 also shows practices located in the Australian Capital Territory or top IRSAD quintile areas had the highest antibiotic prescribing rate in most years. However, practices located in lowest IRSAD quintile areas showed a slight higher frequency in 2017 compared with 2015. Antibiotic prescribing in major cities were 14\% lower in 2017 when compared with 2015, while no significant changes were observed in other areas.

Considering patient characteristics, the frequency of antiviral prescribing was similar in both sexes and although the use of antivirals increased with patient's age in any year, the rise was higher among those aged $<5$ years than in the elderly (68\% and 18\% more frequent in 2017 than 2015, respectively). Antiviral prescribing among Aboriginal and Torres Strait Islanders was approximately $20 \%$ lower compared with other Australians, and this difference remained steady over the period. On the other hand, antiviral prescribing was higher in 2017 than 2015, 
Table 1 ILI consultation rates (per 1000 consultations) according to practices and patients' characteristics. Australia, 20152017

\begin{tabular}{|c|c|c|c|c|}
\hline \multirow[b]{2}{*}{ Variables } & \multicolumn{4}{|c|}{ ILI consultation rates (per 1000 consultations)* } \\
\hline & Total & 2015 & 2016 & 2017 \\
\hline \multicolumn{5}{|l|}{ Practices' characteristics } \\
\hline \multicolumn{5}{|l|}{ State $\dagger$} \\
\hline New South Wales & 3.6 & 2.8 & 2.7 & 5.8 \\
\hline Victoria & 2.6 & 2.4 & 1.7 & 4.1 \\
\hline Queensland & 3.5 & 3.6 & 2.5 & 4.8 \\
\hline Western Australia & 2.2 & 2.3 & 2.5 & 1.7 \\
\hline Tasmania & 3.1 & 2.7 & 2.4 & 4.7 \\
\hline South Australia & 3.0 & 3.0 & 2.2 & 4.4 \\
\hline Australian Capital Territory & 1.7 & 2.0 & 1.0 & 2.4 \\
\hline Northern Territory & 4.3 & 4.2 & 3.1 & 6.4 \\
\hline \multicolumn{5}{|l|}{ Rurality $^{\dagger}$} \\
\hline Major cities & 3.2 & 2.8 & 2.5 & 4.9 \\
\hline Inner regional & 2.6 & 2.4 & 2.1 & 3.7 \\
\hline Outer regional/remote/very remote & 2.7 & 2.8 & 2.2 & 3.3 \\
\hline \multicolumn{5}{|l|}{ IRSAD quintile $^{\dagger}$} \\
\hline Very high & 4.3 & 4.0 & 3.4 & 6.0 \\
\hline High & 2.7 & 2.5 & 2.0 & 4.0 \\
\hline Middle & 3.0 & 2.6 & 2.3 & 4.6 \\
\hline Low & 2.3 & 2.2 & 1.7 & 3.5 \\
\hline Very low & 2.5 & 2.2 & 2.1 & 3.6 \\
\hline \multicolumn{5}{|l|}{ Patients' characteristics } \\
\hline \multicolumn{5}{|l|}{ Gender $^{\dagger}$} \\
\hline Male & 3.3 & 3.0 & 2.6 & 4.6 \\
\hline Female & 2.9 & 2.6 & 2.2 & 4.3 \\
\hline \multicolumn{5}{|l|}{$\mathrm{Age}^{\dagger}$} \\
\hline$<5$ & 2.0 & 1.6 & 1.4 & 3.7 \\
\hline $5-17$ & 4.9 & 4.7 & 3.0 & 7.7 \\
\hline $18-44$ & 4.1 & 3.9 & 3.3 & 5.5 \\
\hline $45-64$ & 3.2 & 2.7 & 2.5 & 4.9 \\
\hline$\geq 65$ & 1.6 & 1.2 & 1.3 & 2.4 \\
\hline \multicolumn{5}{|l|}{ Ethnicity ${ }^{\dagger}$} \\
\hline Non Aboriginal/Torres Strait Islander & 3.0 & 2.7 & 2.3 & 4.4 \\
\hline Aboriginal/Torres Strait Islander & 2.3 & 2.0 & 1.8 & 3.4 \\
\hline Not recorded & 3.2 & 2.9 & 2.5 & 4.8 \\
\hline \multicolumn{5}{|l|}{ IRSAD quintile ${ }^{\dagger}$} \\
\hline Very high & 3.4 & 3.1 & 2.7 & 4.7 \\
\hline High & 2.8 & 2.7 & 2.1 & 4.0 \\
\hline Middle & 3.0 & 2.6 & 2.3 & 4.4 \\
\hline Low & 2.9 & 2.7 & 2.1 & 4.3 \\
\hline Very low & 3.0 & 2.4 & 2.2 & 4.7 \\
\hline \multicolumn{5}{|l|}{ Comorbidities $† \ddagger$} \\
\hline No & 3.4 & 3.1 & 2.6 & 4.8 \\
\hline Yes & 2.4 & 2.0 & 1.8 & 3.7 \\
\hline
\end{tabular}


Table 1 Continued

\begin{tabular}{|c|c|c|c|c|}
\hline \multirow[b]{2}{*}{ Variables } & \multicolumn{4}{|c|}{ ILI consultation rates (per 1000 consultations)* } \\
\hline & Total & 2015 & 2016 & 2017 \\
\hline \multicolumn{5}{|l|}{ Depression/anxiety $^{\dagger}$} \\
\hline Yes & 2.7 & 2.4 & 2.0 & 3.9 \\
\hline Total number of ILI cases & 98086 & 31882 & 27957 & 38306 \\
\hline
\end{tabular}

*Logistic regression models with practices' characteristics are adjusted for each other; logistic regression models with patients' characteristics are adjusted for age, gender and practices' characteristics. Numbers in bold indicate $p$ value $\leq 0.05$ for analyses between the first (reference) category of each variable and the other categories in the same year. $\dagger P$ value $\leq 0.05$ for longitudinal analysis (difference between years in the same category). $\ddagger$ Comorbidities include cardiac disease, chronic neurological disease, chronic respiratory disease, chronic liver disease, immunocompromising conditions and other chronic diseases (including chronic renal failure, diabetes mellitus and haemoglobinopathies). ILI, influenza-like illness; IRSAD, Index of Relative Socioeconomic Advantage and Disadvantage.

independent of the individual's IRSAD quintile, but more than doubled among those in the lowest quintile of socioeconomic disadvantage, reducing the gap with those in the top quintile. Even though the presence of comorbidities did not influence antiviral prescribing, it was slightly lower among those with a mental health problem in any of the investigated years.

Antibiotic prescribing was more frequent among women, in the elderly, among those with some comorbidity or mental health illness, but was not substantially influenced by ethnicity or an individual's IRSAD. Although most groups showed a lower frequency of antibiotic prescribing in 2017 than 2015, it remained steady among individuals aged $<5$ years or those in the lowest IRSAD quintile.

Figure 2 shows that having a respiratory or neurological chronic condition was related to a higher frequency of antiviral prescription for the management of ILI in 2017. Regarding antibiotics, patients with chronic respiratory conditions were also more likely to be managed with these medications than their peers or than patients affected by another chronic disease, and this pattern was similar across the period.

In summary, characteristics of the practice were more strongly associated with ILI diagnosis (pseudo $\mathrm{R}^{2}=3 \%$ ), antiviral (pseudo $\mathrm{R}^{2}=9 \%$ ) and antibiotic prescribing (pseudo $\mathrm{R}^{2}=2 \%$ ) than patients' characteristics (pseudo $\mathrm{R}^{2}<1 \%$ for all of them).

\section{DISCUSSION}

Using a large dataset of over four million patients and 32 million consultations, we investigated the epidemiology of ILI in Australian general practice between 2015 and 2017, as well as changes in the prescription of antivirals and antibiotics for its treatment. Four main findings can be highlighted. First, ILI was more frequent in 2017 than previous years. Second, antibiotic prescribing declined across the three years, while, the use of antivirals increased and in 2017 was higher than the former. Third, practice characteristics were strongly associated with ILI diagnosis and management. Practices located in higher socioeconomic areas not only had higher ILI consultation rates, they also prescribed more antibiotics and antivirals than practices located in lower socioeconomic areas. Finally, the presence of a chronic medical condition or mental health problem was associated with lower ILI consultation rates. This is likely explained by the fact that such people attend more frequently for other reasons than 'healthy' individuals, thus inflating the denominator and leading to lower rates. This is a characteristic inherent to this measurement, which could lead to the misconception that ILI is less frequent among patients affected by chronic comorbidities, when they are actually more susceptible to develop ILI and its complications. ${ }^{27} 28$ In fact, antibiotic prescribing was higher among these patients compared with 'healthy' participants, although the use of antivirals was similar. Moreover, individuals with a chronic respiratory condition were more likely to be managed with antivirals or antibiotics than individuals with other comorbidities or patients with no chronic or mental health condition.

Peaks for ILI occurring in weeks 33-36 are consistent with results from the Australian Sentinel Practices Research Network ${ }^{19} 20$ (a mixed online and data extraction system with over 250 GPs across Australia), FluTracking $^{31}$ (an online system based on patients' self-reported data) and the National Notifiable Diseases Surveillance System ${ }^{32}$ (influenza notifications confirmed by laboratory). According to all these sources, ILI consultation rates in 2017 were the highest for the 3-year period, reflecting a longer duration and more intense season in that year. Moreover, consistent with our results, all these surveillance systems identified lower ILI consultation rates in Western Australia, especially in 2017. ${ }^{19} 3132$

To our knowledge, this is the first Australian study describing the prescription of antivirals for the management of ILI cases, and only a few studies have investigated this topic outside the USA. ${ }^{21}{ }^{22}$ A study conducted in the 
Table 2 Prescribing patterns of antivirals and antibiotics for ILI according to general practices and patients' characteristics, Australia, 2015-2017

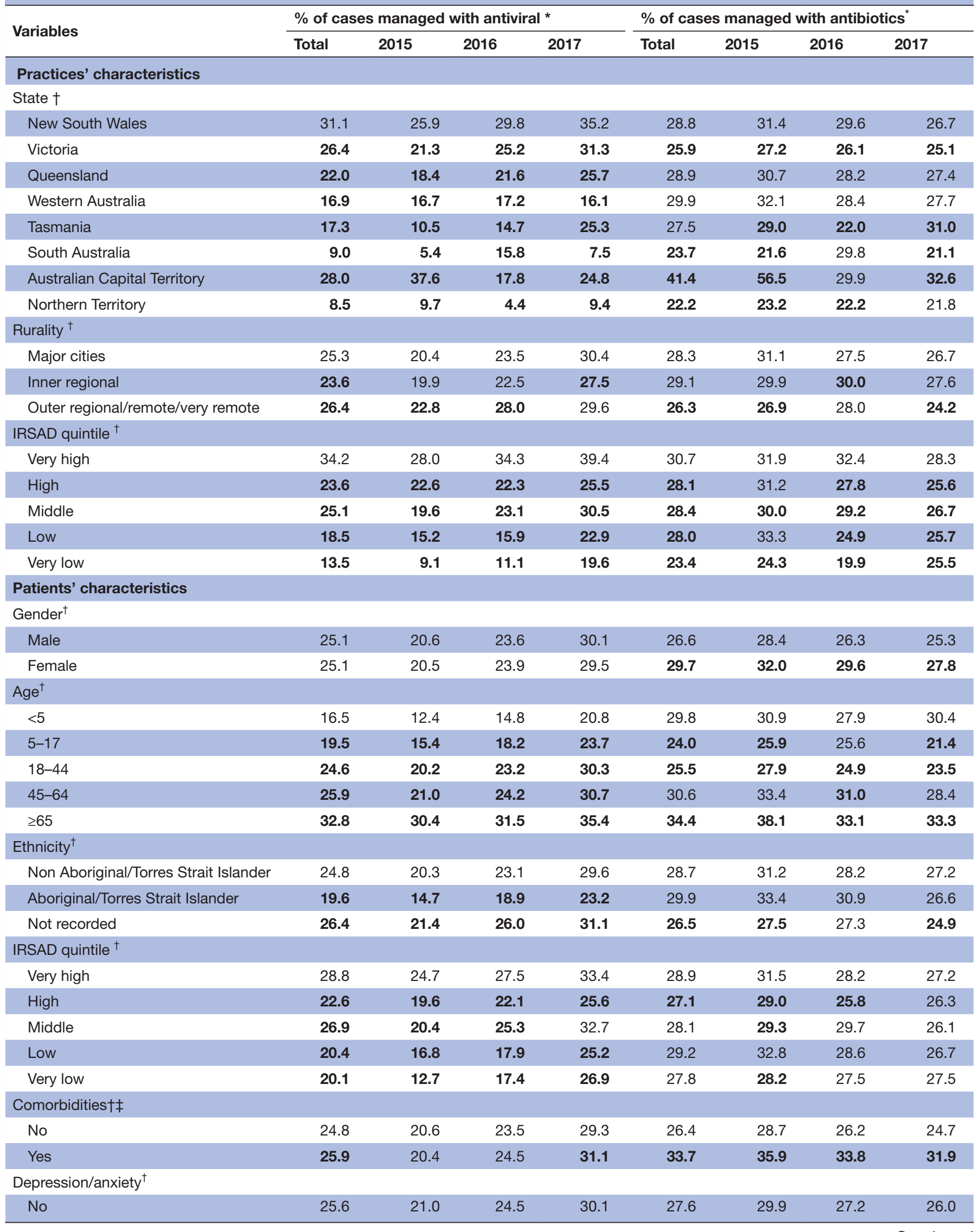


Table 2 Continued

\begin{tabular}{|c|c|c|c|c|c|c|c|c|}
\hline \multirow{2}{*}{ Variables } & \multicolumn{4}{|c|}{$\%$ of cases managed with antiviral * } & \multicolumn{4}{|c|}{$\%$ of cases managed with antibiotics ${ }^{*}$} \\
\hline & Total & 2015 & 2016 & 2017 & Total & 2015 & 2016 & 2017 \\
\hline Yes & 23.7 & 19.2 & 21.6 & 28.9 & 30.1 & 31.7 & 30.4 & 28.6 \\
\hline Total & 25.0 & 20.6 & 23.7 & 29.7 & 28.3 & 30.3 & 28.0 & 26.7 \\
\hline
\end{tabular}

'Logistic regression models with practices' characteristics are adjusted for each other; logistic regression models with patients' characteristics are adjusted for age, gender and practices' characteristics. Numbers in bold indicate $p$ value $\leq 0.05$ for analyses between the first (reference) category of each variable and the other categories in the same year.

$\dagger P$ value $\leq 0.05$ for longitudinal analysis (difference between years in the same category).

$\ddagger$ Comorbidities include: cardiac disease, chronic neurological disease, chronic respiratory disease, chronic liver disease, immunocompromising conditions and other chronic diseases (including chronic renal failure, diabetes mellitus or haemoglobinopathies). ILI, influenza-like illness; IRSAD, Index of Relative Socioeconomic Advantage and Disadvantage.

Netherlands with data from electronic medical records of a representative sample of general practices found only $5.5 \%$ of all patients diagnosed with ILI were prescribed antivirals, and although that percentage was similar in both sexes, it was more frequently used in the elderly, among children under the age of twoyears or patients with comorbidities. ${ }^{22}$ In the USA, a study using data from outpatients sites associated with the US Influenza Vaccine Effectiveness Network explored antiviral prescribing in electronic medical records of patients at high-risk of complications and showed a non-linear increase over time, changing from $2 \%$ in $2011 / 2012$ to $8.9 \%$ in

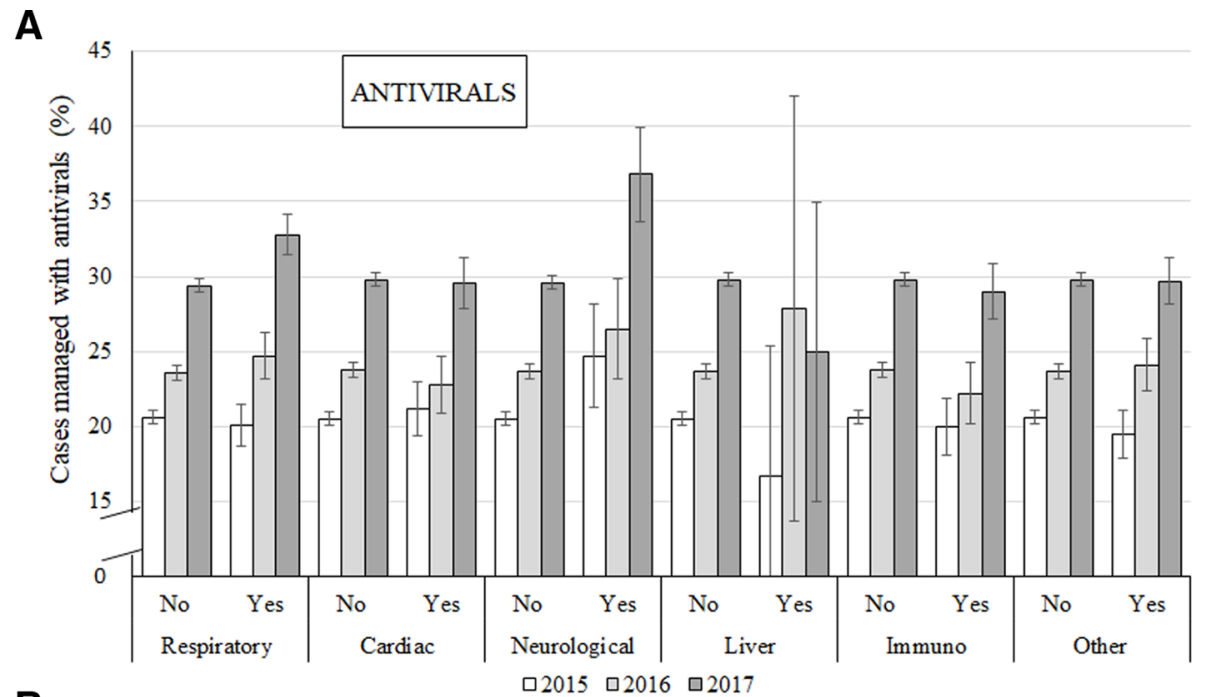

B

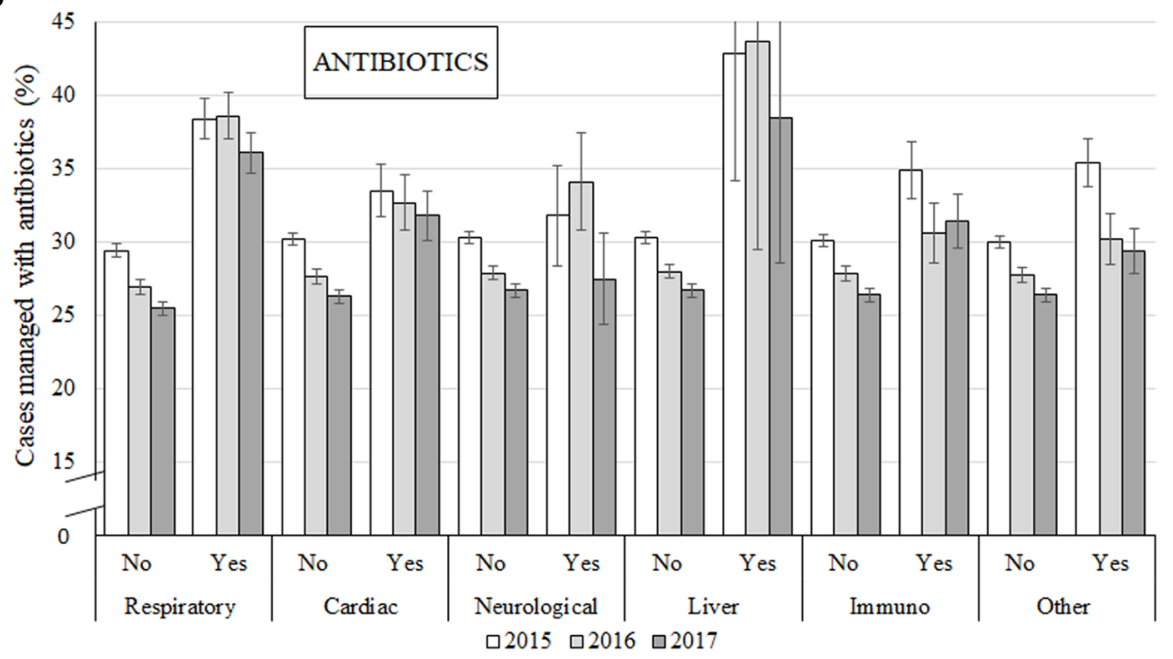

Figure 2 Prescribing patterns (antivirals or antibiotics) for influenza-like illness in patients with chronic respiratory, cardiac, neurological or liver disease, immunocompromising conditions or other chronic diseases (including chronic renal failure, diabetes mellitus or haemoglobinopathies), Australia 2015-2017. 
2012/2013 and 4.7\% in 2015/2016. ${ }^{33}$ Another American investigation using data from the same source but including people with or without risk factors, performed during the influenza seasons of 2013 to 2015 found $20 \%$ of patients with laboratory-confirmed influenza received an antiviral prescription and $29 \%$ were managed with antibiotics. Moreover, $3 \%$ of them were managed with both medications at the same time (compared with 8.2\% in our study). ${ }^{12}$ In Australia, although some studies have shown a reduction in antibiotic prescribing in the recent years, ${ }^{3435}$ estimates suggest their use for acute non-bacterial respiratory infections is still four to nine times more frequent than necessary. ${ }^{15}$ We also found antibiotic prescription for ILI treatment was slightly higher in women, which is consistent with findings from a large Sweden study, ${ }^{36}$ but studies conducted in the US and England found no gender differences. ${ }^{12} 2937$

According to our findings, the frequency of antiviral prescribing for ILI management was $44 \%$ higher (in relative terms) in 2017 than in 2015, regardless of a practice's region or socioeconomic status, while antibiotic prescribing was slightly lower by the end of the investigated period. However, practices located in high-population states (ie, New South Wales and Victoria) or top IRSAD quintile areas prescribed antivirals up to three times more frequently than practices from less populated states or lower IRSAD quintile areas. Moreover, practice characteristics were more important determinants of antiviral prescribing than patients' characteristics, as some of the associations with individual variables were null (ie, gender, presence of comorbidities or mental health problems) or less evident (ie, patient's IRSAD quintile). Further studies will be necessary to explore if the reasons behind the difference between antiviral and antibiotic prescribing are inherent to practice (eg, geographic characteristics, complexity of the practice, volume of patients), provider (eg, training, perception of patients' needs), the severity of the influenza season with attendant publicity and department of health warnings or other external factors (eg, state recommendations/policies). ${ }^{38}{ }^{39}$ In any case, it seems that the differences in antibiotic prescribing between socioeconomic groups has reduced while that for antivirals has increased and is replicating disparities previously reported for antibiotics in other countries (ie, more frequent prescribing in higher socioeconomic areas and among whites compared with other ethnic groups) ${ }^{40-42}$ Such results should be considered carefully, as correlations between socioeconomic status and medication prescription are highly context-dependent, which could explain the contrasting results identified in different countries. ${ }^{39} 4344$

The increasing use of antivirals in all age groups is noteworthy, as one-fifth of children with an ILI are currently treated with these medications. Studies on the effectiveness of antivirals for the management of ILI in healthy individuals or children are low quality, and although some of them have suggested they reduce the risk of complications (ie, ear infections and pneumonia), the only consistent benefit identified in systematic reviews has been a small reduction in symptoms duration ( 1 day).$^{56}$ Therefore, managing ILI with antivirals can be questioned and only used after an assessment of the patient's risk of complications, potential health benefits, possible side effects (eg, digestive symptoms, headache, renal complications, psychiatric events) and health costs, ${ }^{5}$ which has already been explored in other countries. ${ }^{21}{ }^{22}$ Vaccination and preventive non-pharmaceutical measures, such as case isolation, hand-washing or the use of masks may be more cost-effective strategies to tackle the transmission of influenza among healthy people. ${ }^{45}$

Even though antivirals were more frequently used in the elderly, the prescription of these drugs was not more frequent among those affected by chronic conditions (ie, respiratory, cardiac, neurological, hepatic, immunosuppressive or other metabolic conditions). Nonetheless, antibiotic prescription for ILI was more frequent among those with some comorbidity, which is consistent with results from studies conducted in England and Sweden. ${ }^{3637}$ Therefore, according to our findings, antiviral use in Australian general practice does not seem to be targeting individuals who could benefit most from reduced hospitalisations and mortality due to influenza complications. ${ }^{6}{ }^{46}$ However, a different pattern was observed for those affected by chronic respiratory conditions, as the prescription of antivirals and antibiotics were both more frequent among these group of patients. This could be explained as a consequence of increased respiratory symptoms and physical exam findings among patients with chronic respiratory conditions affected by influenza, as a GPs' decision to prescribe antibiotics has been found to be influenced by the severity of the patient's illness. ${ }^{143647}$

\section{Strengths and limitations}

The study has significant strengths; a large sample of patients, including individuals of all age groups, ethnicity or sex, and practices from all Australian states, socioeconomic areas or remoteness. The observed pattern for ILI consultation rates within and over the years in our results, moreover, is consistent with findings from other Australian surveillance systems using different data sources. ${ }^{19} 3132$ Nevertheless, the study has some limitations that have to be recognised. Although the MedicineInsight programme reflects real clinical practice, with diagnoses and treatment decisions recorded by GPs, the quality, accuracy and completeness of recording may vary between GPs, since the information can be registered as free text instead of using standardised medical codes. Also, strategies used for data extraction have not been validated, which could affect the accuracy of the information (under or overestimate) ${ }^{23}$ However, although some under-reporting of clinical information (diagnosis or reason for encounter) is expected due to these possible limitations, they do not explain the associations identified or the consistency of the ILI results compared with other surveillance systems. Finally, the selection of practices by MedicineInsight uses a non-random technique, so sampling bias cannot be eliminated. However, the sample distribution according to sociodemographic characteristics is comparable with the last Australian census. ${ }^{1748}$ 


\section{CONCLUSION}

Results using ILI data from the NPS MedicineInsight database is consistent with other Australian sources. Antibiotic prescribing for ILI was lower in 2017 than in previous years, while the use of antivirals has risen during the same period, results that may be confirmed as a trend with data from future years. Despite the more frequent use of antivirals, their use is not targeted at people with a high risk of complications. Australian researchers, policy-makers and organisations have been discussing a national strategy for reducing antibiotic usage. ${ }^{1349}{ }^{50}$ According to our findings, they should also monitor the use of antivirals for the management of ILI in Australian general practice. Strategies would require multifaceted approaches involving physicians, patients and public education, novel interventions, adequate resources and a sustained effort to improve health outcomes, reduce health costs, minimise adverse side effects and antimicrobial resistance.

Acknowledgements The authors acknowledge NPS MedicineWise for their support in the development of this research.

Contributors All authors made significant contributions to the manuscript and are responsible for its content. NS conceived the idea and planned this study. CDOB and DG-C were responsible for data extraction and analysis, interpreting and presenting the results. CDOB wrote the first draft and the revisions. DG-C contributed to the manuscript refinement. NS contributed to revisions and the final version of the manuscript. All authors have read and approved the final manuscript.

Funding The authors have not declared a specific grant for this research from any funding agency in the public, commercial or not-for-profit sectors.

Competing interests None declared.

Patient consent for publication Not required.

Ethics approval The Human Research Ethics Committee of the University of Adelaide exempted this study of an ethical review, considering it used existing and non-identifiable data.

Provenance and peer review Not commissioned; externally peer reviewed.

Data sharing statement No additional data are available, as the original dataset belongs to a third party.

Open access This is an open access article distributed in accordance with the Creative Commons Attribution Non Commercial (CC BY-NC 4.0) license, which permits others to distribute, remix, adapt, build upon this work non-commercially, and license their derivative works on different terms, provided the original work is properly cited, appropriate credit is given, any changes made indicated, and the use is non-commercial. See: http://creativecommons.org/licenses/by-nc/4.0/.

\section{REFERENCES}

1. World Health Organization (WHO). Influenza (Seasonal). $2018 \mathrm{http} / / /$ www.who.int/en/news-room/fact-sheets/detail/influenza-(seasonal) (Accessed on 10 Jul 2018).

2. Australian Bureau of Statistics (ABS). Australia's leading causes of death. 2016 http://www.abs.gov.au/ausstats/abs@.nsf/Lookup/ by\%20Subject/3303.0 2016 Main\%20Features Australia's\%20 leading\%20causes\%20of\%20death,\%202016 3 (Accessed on 10 Jul 2018).

3. Australian Government. Department of Health, Australian Health Management Plan for Pandemic Influenza. 2014 http://www.health. gov.au/internet/main/publishing.nsf/content/ohp-ahmppi.htm (Accessed on $10 \mathrm{Jul}$ 2018).

4. Anikeeva O, Braunack-Mayer AJ, Street JM. How will Australian general practitioners respond to an influenza pandemic? A qualitative study of ethical values. Med J Aust 2008;189:148-50.

5. Jefferson T, Jones MA, Doshi P, et al. Neuraminidase inhibitors for preventing and treating influenza in adults and children. Cochrane Database Syst Rev 2014;19.

6. Hsu J, Santesso N, Mustafa R, et al. Antivirals for treatment of influenza: a systematic review and meta-analysis of observational studies. Ann Intern Med 2012;156:512-24.
7. Muthuri SG, Venkatesan S, Myles PR, et al. Effectiveness of neuraminidase inhibitors in reducing mortality in patients admitted to hospital with influenza A H1N1pdm09 virus infection: a meta-analysis of individual participant data. Lancet Respir Med 2014;2:395-404.

8. Harris AM, Hicks LA, Qaseem A. Appropriate antibiotic use for acute respiratory tract infection in adults: advice for high-value care from the american college of physicians and the centers for disease control and prevention. Ann Intern Med 2016;164:425-34.

9. Antibiotic Expert Groups. Therapeutic Guidelines: Antibiotic. Melbourne: Therapeutic Guidelines Limited, 2014.

10. Centers for Disease Control and Prevention (CDC). Antibiotic resistance threats in the United States. 2013 http://www.cdc.gov/ drugresistance/threat-report-2013/ (Accessed on $10 \mathrm{Jul}$ 2018).

11. Fleming-Dutra KE, Hersh AL, Shapiro DJ, et al. Prevalence of inappropriate antibiotic prescriptions among US Ambulatory Care Visits, 2010-2011. JAMA 2016;315:1864-73.

12. Havers FP, Hicks LA, Chung JR, et al. Outpatient antibiotic prescribing for acute respiratory infections during influenza seasons. JAMA Netw Open 2018;1:e180243.

13. Del Mar CB, Scott AM, Glasziou PP, Hoffmann T, et al. Reducing antibiotic prescribing in Australian general practice: time for a national strategy. Med J Aust 2017;207:401-6.

14. Pouwels KB, Dolk FCK, Smith DRM, et al. Actual versus 'ideal' antibiotic prescribing for common conditions in English primary care. J Antimicrob Chemother 2018;73(suppl 2):19-26.

15. McCullough AR, Pollack AJ, Plejdrup Hansen M, et al. Antibiotics for acute respiratory infections in general practice: comparison of prescribing rates with guideline recommendations. Med $J$ Aust 2017;207:65-9.

16. González-Chica DA, Vanlint S, Hoon E, et al. Epidemiology of arthritis, chronic back pain, gout, osteoporosis, spondyloarthropathies and rheumatoid arthritis among 1.5 million patients in Australian general practice: NPS MedicineWise Medicinelnsight dataset. BMC Musculoskelet Disord 2018;19:20.

17. Medicinelnsight. Medicinelnsight Data Book version 1.1. Sydney: NPS MedicineWise, February. 2018 https://cdn0.scrvt.com/ 08ab3606b0b7a8ea53fd0b40b1c44f86/475dedf8d41a8a72/ 88fd0674e192/Medicinelnsight-Data-Book-and-Dictionary_v1.1.pdf (Accessed on 10 Aug 2018).

18. Halliday L, Roberts L, Hampson A. Annual report of the National Influenza Surveillance Scheme, 1998. Commun Dis Intell 1999;23:185-92.

19. Australian Sentinel Practices Research Network (ASPREN). Syndromic Surveillance Results. 2018 https://aspren.dmac.adelaide. edu.au/ (Accessed on 12 Jul 2018).

20. Varghese BM, Dent E, Chilver M, et al. Epidemiology of viral respiratory infections in Australian working-age adults (20-64 years): 2010-2013. Epidemiol Infect 2018;146:619-26.

21. Hardelid P, Rait G, Gilbert R, et al. Prescribing of neuraminidase inhibitors for influenza in UK primary care since the 2009 pandemic. Epidemiol Infect 2016;144:772-6.

22. Hooiveld M, van de Groep T, Verheij TJ, et al. Prescription of antiviral drugs during the 2009 influenza pandemic: an observational study using electronic medical files of general practitioners in the Netherlands. BMC Pharmacol Toxicol 2013;14:55.

23. Benchimol El, Smeeth L, Guttmann A, et al. The REporting of studies Conducted using Observational Routinely-collected health Data (RECORD) statement. PLoS Med 2015;12:e1001885.

24. Tu K, Manuel D, Lam K, et al. Diabetics can be identified in an electronic medical record using laboratory tests and prescriptions. $J$ Clin Epidemiol 2011;64:431-5.

25. Woodfield R, Grant I, Sudlow CL. Accuracy of Electronic Health Record Data for Identifying Stroke Cases in Large-Scale Epidemiological Studies: A Systematic Review from the UK Biobank Stroke Outcomes Group. PLoS One 2015;10:e0140533.

26. Australian Bureau of Statistics (ABS). Geography. http://www.abs. gov.au/geography (Accessed on 31 Jul 2018).

27. Nokleby H, Nicoll A. Risk groups and other target groups Preliminary ECDC guidance for developing influenza vaccination recommendations for the season 2010-11. 2010;15:pii=19525.

28. Vestbo J, Hurd SS, Agustí AG, et al. Global strategy for the diagnosis, management, and prevention of chronic obstructive pulmonary disease: GOLD executive summary. Am J Respir Crit Care Med 2013;187:347-65.

29. Mehta N, Schilder A, Fragaszy E, et al. Antibiotic prescribing in patients with self-reported sore throat. J Antimicrob Chemother 2017;72:914-22.

30. Little P, Dorward M, Warner G, et al. Importance of patient pressure and perceived pressure and perceived medical need for investigations, referral, and prescribing in primary care: nested observational study. BMJ 2004;328:444. 
31. FluTracking. FluTracking Reports (Australia). https://info.flutracking. net/reports-2/australia-reports/ (Accessed on 30 Jul 2018).

32. Australian Government. Department of Health. National Notifiable Diseases Surveillance System. 2018. Available at http://www9.health. gov.au/cda/source/rpt_3.cfm (Accessed on 30/07/2018).

33. Stewart RJ, Flannery B, Chung JR, et al. Influenza Antiviral Prescribing for Outpatients With an Acute Respiratory Illness and at High Risk for Influenza-Associated Complications During 5 Influenza Seasons-United States, 2011-2016. Clin Infect Dis 2018;66:1035-41.

34. Pan Y, Henderson J, Britt H. Antibiotic prescribing in Australian general practice: how has it changed from 1990-91 to 2002-03? Respir Med 2006;100:2004-11.

35. Britt $\mathrm{H}$, Harrison $\mathrm{C}$, Miller G. The real story, GP prescribing of antibiotics for respiratory tract infections - from BEACH. FMRC, University of Sydney 2012

36. Ternhag A, Grünewald M, Nauclér P, et al. Antibiotic consumption in relation to socio-demographic factors, co-morbidity, and accessibility of primary health care. Scand J Infect Dis 2014;46:888-96.

37. Smith DRM, Dolk FCK, Smieszek T, et al. Understanding the gender gap in antibiotic prescribing: a cross-sectional analysis of English primary care. BMJ Open 2018;8:e020203.

38. Gaur AH, Hare ME, Shorr RI. Provider and practice characteristics associated with antibiotic use in children with presumed viral respiratory tract infections. Pediatrics 2005;115:635-41.

39. Wise J. Antibiotic prescribing is higher in deprived areas of England. BMJ 2015;351:h6117.

40. Dallas A, Magin P, Morgan S, et al. Antibiotic prescribing for respiratory infections: a cross-sectional analysis of the ReCEnT study exploring the habits of early-career doctors in primary care. Fam Pract 2015;32:49-55.
41. Goyal MK, Johnson TJ, Chamberlain JM, et al. Racial and Ethnic Differences in Antibiotic Use for Viral Illness in Emergency Departments. Pediatrics 2017;140:e20170203.

42. Yaeger JP, Temte JL, Hanrahan LP, et al. Roles of Clinician, Patient, and Community Characteristics in the Management of Pediatric Upper Respiratory Tract Infections. Ann Fam Med 2015;13:529-36.

43. Wang KY, Seed P, Schofield P, et al. Which practices are high antibiotic prescribers? A cross-sectional analysis. Br J Gen Pract 2009;59:e315-e320.

44. Covvey JR, Johnson BF, Elliott V, et al. An association between socioeconomic deprivation and primary care antibiotic prescribing in Scotland. J Antimicrob Chemother 2014;69:835-41.

45. Jefferson T, Del Mar CB, Dooley L, Ferroni E, et al. Physical interventions to interrupt or reduce the spread of respiratory viruses. Cochrane Database Syst Rev 2011;141:CD006207.

46. Van Kerkhove MD, Vandemaele KA, Shinde V, et al. Risk factors for severe outcomes following 2009 influenza A (H1N1) infection: a global pooled analysis. PLoS Med 2011;8:e1001053.

47. McKay R, Mah A, Law MR, et al. Systematic review of factors associated with antibiotic prescribing for respiratory tract infections. Antimicrob Agents Chemother 2016;60:4106-18.

48. Australian Bureau of Statistics (ABS). Table Builder. https://guest. censusdata.abs.gov.au/webapi/jsf/tableView/tableView.xhtml (Accessed on 31 Jul 2018).

49. Arnold SR, Straus SE. Interventions to improve antibiotic prescribing practices in ambulatory care. Cochrane Database Syst Rev 2005;4:Cd003539.

50. Magin P, Tapley A, Morgan S, et al. Reducing early career general practitioners' antibiotic prescribing for respiratory tract infections: a pragmatic prospective non-randomised controlled trial. Fam Pract 2018;35:53-60. 